\title{
Cold- and light-induced changes in the transcriptome of wheat leading to phase transition from vegetative to reproductive growth Mark O Winfield ${ }^{* \dagger 1}$, Chungui Lu ${ }^{\dagger 2}$, Ian D Wilson ${ }^{3}$, Jane A Coghill ${ }^{1}$ and Keith J Edwards ${ }^{1}$
}

Address: ${ }^{1}$ School of Biological Sciences, University of Bristol, Bristol, BS8 1UG, UK, 2 School of Science and Technology, Nottingham Trent University, Nottingham, UK and ${ }^{3}$ School of Life Sciences, University of the West of England, Frenchay Campus, Coldharbour Lane, Bristol, BS16 1QY, UK

Email: Mark O Winfield* - Mark.Winfield@bristol.ac.uk; Chungui Lu - chungui.lu@ntu.ac.uk; Ian D Wilson - Ian2.Wilson@uwe.ac.uk; Jane A Coghill - Jane.Coghill@bristol.ac.uk; Keith J Edwards - K.J.Edwards@bristol.ac.uk

* Corresponding author †Equal contributors

Published: II May 2009

BMC Plant Biology 2009, 9:55 doi:10.1 I86/147|-2229-9-55
Received: 26 October 2008

Accepted: II May 2009

This article is available from: http://www.biomedcentral.com/I47I-2229/9/55

(C) 2009 Winfield et al; licensee BioMed Central Ltd.

This is an Open Access article distributed under the terms of the Creative Commons Attribution License (http://creativecommons.org/licenses/by/2.0), which permits unrestricted use, distribution, and reproduction in any medium, provided the original work is properly cited.

\begin{abstract}
Background: For plants to flower at the appropriate time, they must be able to perceive and respond to various internal and external cues. Wheat is generally a long-day plant that will go through phase transition from vegetative to floral growth as days are lengthening in spring and early summer. In addition to this response to day-length, wheat cultivars may be classified as either winter or spring varieties depending on whether they require to be exposed to an extended period of cold in order to become competent to flower. Using a growth regime to mimic the conditions that occur during a typical winter in Britain, and a microarray approach to determine changes in gene expression over time, we have surveyed the genes of the major pathways involved in floral transition. We have paid particular attention to wheat orthologues and functional equivalents of genes involved in the phase transition in Arabidopsis. We also surveyed all the MADS-box genes that could be identified as such on the Affymetrix genechip wheat genome array.
\end{abstract}

Results: We observed novel responses of several genes thought to be of major importance in vernalisation-induced phase transition, and identified several MADS-box genes that might play an important role in the onset of flowering. In addition, we saw responses in genes of the Gibberellin pathway that would indicate that this pathway also has some role to play in phase transition.

Conclusion: Phase transition in wheat is more complex than previously reported, and there is evidence that day-length has an influence on genes that were once thought to respond exclusively to an extended period of cold.

\section{Background}

In plants, the timing of the change from vegetative to reproductive growth is critical for successful reproduction, and must occur when both internal and external conditions are appropriate. The environmental cues of daylength and temperature have a strong influence on flower- ing, and the ability to perceive and respond to these cues is controlled through the photoperiod and vernalisation pathways, respectively [1].

Wheat (Triticum aestivum L.) is normally a long-day plant, flowering in spring and early summer when days are 
lengthening [2]. Additionally, wheat cultivars can be broadly divided into two categories, winter or spring, according to whether they require an extended period of cold to become competent to flower. In winter varieties, change from vegetative to reproductive phase is promoted by exposure to low temperatures $\left(3^{\circ} \mathrm{C}-8^{\circ} \mathrm{C}\right)$ for $4-6$ weeks. These varieties are planted in the autumn so that seedlings are exposed to the cold of winter and so become competent to flower. However, they only become committed to flower as days lengthen in the spring. In addition to these two external factors, different wheat varieties can be distinguished by the intrinsic rate at which they tend to pass from floral induction to heading. This tendency is referred to as earliness per se [3].

In Arabidopsis, the genetic factors underpinning phase change have been well-characterised [4-6]. Four major genetic pathways regulate this transition: the photoperiod and vernalisation pathways mediate responses to the environmental cues of light and cold, respectively, whilst the autonomous and gibberellin pathways are dependent on endogenous signals [7-10]. Unfortunately, in studying phase transition in cereals one cannot draw directly on the information gained from the study of Arabidopsis since orthologues can't always be found. For example, cereals do not possess an orthologue of the Arabidopsis FLOWERING LOCUS C (AtFLC) gene, an important repressor of flowering $[11,12]$. To confuse matters more, genes with similar sequence don't necessarily have the same function. None-the-less, comparative genetics may still provide a promising starting point for a search of candidate genes involved in phase transition in the cereals. This certainly seems to be the case for the photoperiod pathway where there is a remarkable degree of conservation of functional components between Arabidopsis and rice [13]. However, the vernalisation pathway may have evolved independently in dicots and monocots such that they use different genes to retard flowering until winter has passed [8]. Thus, although much is known about the genes involved in floral transition in Arabidopsis, only recently have candidates for the key regulators determining vernalisation requirement in cereals been identified $[14,15]$.

In the temperate cereals barley and wheat, two genes, $V R N 1$ and VRN2 (unrelated to the identically named genes in Arabidopsis), have been reported to be the key elements in the vernalisation pathway [15-17]. Several papers addressing the issue of vernalisation have considered the interaction of just these two genes. A case in point is the model presented by Yan et al. [15] in which TaVRN1 and TaVRN2 are presented, respectively, as a promoter and repressor of flowering (see Additional file 1, for a schematic representation of this). According to the model, TaVRN1 is constitutively expressed in spring wheats but in winter varieties is up-regulated as a consequence of ver- nalisation. Conversely, TaVRN2 is highly expressed in winter varieties, thus repressing flowering, but not in spring varieties. It has been hypothesised that in winter wheats, extended periods of cold bring about down-regulation of TaVRN2 and, as a consequence, the up-regulation of TaVRN1 and commitment to flowering.

Using Affymetrix Genechip Wheat Genome Arrays as the platform for our analysis, we were able to consider the broad-scale response of the transcriptome to the changes in temperature and light that occur during a simulated autumn to winter transition. In this paper, however, we principally focus our attention on the components of the vernalisation pathway. However, our experimental design was such that we have also been able to draw conclusions about the impact on phase transition of several components of other flowering pathways. Finally, using a microarray approach rather than a more targeted approach allowed us to observe the expression profiles of genes that, a priori, we would not have assayed, and have been able to identify potentially important new players in phase transition.

\section{Results and discussion}

The growth conditions were established to broadly simulate those that would be experienced by plants sown in October in Britain. In particular, attention was paid to the details of suitable light quality and photoperiod (Table 1), because there is evidence that sustaining high light intensity and an extended photoperiod while reducing temperature may result in stress related patterns of gene expression [18].

Winter (Harnesk and Solstice) and spring wheat (Paragon) varieties of wheat were grown at $16^{\circ} \mathrm{C}$ day $/ 14^{\circ} \mathrm{C}$ night for 21 days, and then, over a period of nine weeks, exposed to a slow, stepped decline in temperature and light (Table 1). The developmental state of the crown tissue was assessed in thin tissue sections at the end of each temperature stage (every 7 days from the third week onwards). As shown in Figure 1, after six weeks' growth, crowns in the spring variety were much more advanced in their development than those in the two winter varieties while the apices of the winter varieties were at approximately stage 2 (elongation and early formation of leaf primordia), according to the scale proposed by Gardner et al. [19], in Paragon a distinct spike with enlarging spikelets and early glumes was evident (stage 5 or 6 ). Indeed, apices of Paragon showed signs of double ridge formation as early as the fifth week. The winter varieties were assessed to be fully "vernalised" 12 weeks post-germination, and plants treated in this way went on to flower when returned to warmer, long-day conditions (see Additional file 2). Unvernalised, plants of Harnesk and Solstice did not flower (see Additional file 2, A). 
Table I: Growth condition for the time-course experiment (PAR = photosynthetically active radiation).

\begin{tabular}{|c|c|c|c|c|c|}
\hline \multirow{2}{*}{$\begin{array}{c}\text { Duration } \\
\text { (days) }\end{array}$} & \multirow[t]{2}{*}{ Weeks after germination } & \multicolumn{2}{|c|}{ Temperature $\left({ }^{\circ} \mathrm{C}\right)$} & \multirow[t]{2}{*}{ Day-length (h) } & \multirow[t]{2}{*}{ PAR } \\
\hline & & Day & Night & & \\
\hline 21 & 3 & 16 & 14 & 14 & 280 \\
\hline 7 & 4 & 14 & 12 & 14 & 280 \\
\hline 7 & 5 & 14 & 10 & 12 & 185 \\
\hline 7 & 6 & 12 & 10 & 11 & 185 \\
\hline 7 & 7 & 12 & 8 & 11 & 185 \\
\hline 7 & 8 & 10 & 6 & 10 & 95 \\
\hline 7 & 9 & 8 & 4 & 9 & 95 \\
\hline 7 & 10 & 6 & 2 & 8 & 95 \\
\hline 7 & 11 & 4 & 2 & 8 & 95 \\
\hline 7 & 12 & 2 & 2 & 8 & 48 \\
\hline
\end{tabular}

Global gene expression profiles showed there to be differences between the three varieties, between the two tissues (crown and leaf) and across the time course of the experiment (GEO accession number for array data is GSE11774). The most marked difference in gene expression was between leaf and crown (Figure 2) with 22.8 $28.4 \%$ of the transcripts being differentially expressed (Table 2). In contrast, pair-wise comparisons between the cultivars at the end of three weeks, at which time all plants had received exactly the same treatment, showed only 1.5 $-3.7 \%$ of transcripts in crown tissue and $1.3-2.8 \%$ in leaf tissue to be differentially expressed (Table 3 ).

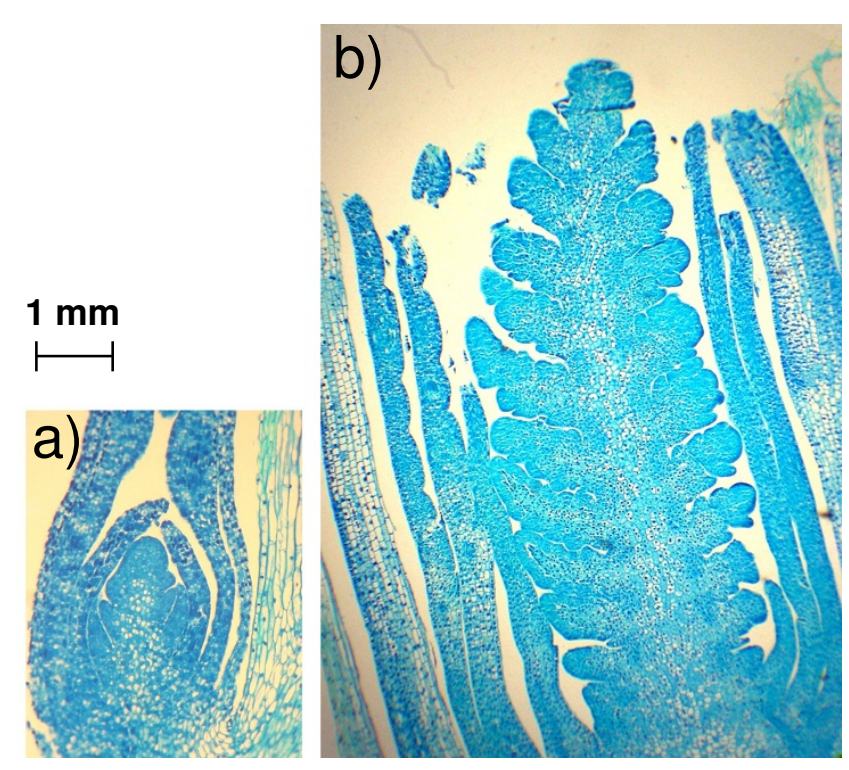

Figure I

Dissected crown tissue from 6 week old plants that have experienced a gradual decline in temperature and light: a) Solstice, a winter variety; b) Paragon, a spring variety. The two images are at the same magnification.
In both tissues, the transcripts of thousands of genes showed statistically significant changes in abundance across the time-course. However, these global patterns do not interest us in this paper. Instead, we focus on the dynamics of expression of genes involved in phase transition. That is, initially we consider the small number of genes reported to be specifically involved in vernalisation in wheat, and then briefly focus on reported orthologues and functional equivalents of the components of the other three pathways involved in phase transition in Arabidopsis. Finally, we consider all those features annotated as MADS-box genes.

\section{Vernalisation in wheat}

In our study, the profiles of transcript abundance of TaVRN1, the proposed major promoter of flowering, were entirely consistent with those reported in other studies $[15,20,21]$. At three weeks, prior to exposure to cold, TaVRN1 transcript was much more abundant in Paragon than in the two winter varieties (Figure 3a). In Paragon, transcript levels remained high across the time-course. In the winter varieties, transcript levels for TaVRN1 were initially low but by 9 weeks had increased, and by 12 weeks, when plants were assessed to be fully vernalised (plants transferred to long days at $16^{\circ} \mathrm{C}$ went on to flower - see Additional file 2, B), there had been an approximately 10 fold increase in abundance. These patterns, confirmed by qRT-PCR (Pearson Correlation Coefficient $=0.76$ ), are

Table 2: The number of statistically significant differences in gene expression between crown and leaf.

\begin{tabular}{lccc}
\hline & \multicolumn{3}{c}{ Crown vs Leaf } \\
\cline { 2 - 4 } & Harnesk & Paragon & Solstice \\
\hline 2 Fold & 17427 & 13977 & 14501 \\
5 Fold & 6129 & 5277 & 5394 \\
\hline
\end{tabular}

Values derived from a volcano plot of 2 and 5 fold differences with ttest $p$ value of 0.05 . 
Table 3: The number of statistically significant differences between cultivars three weeks post germination.

\begin{tabular}{lcccccc}
\hline & \multicolumn{2}{c}{ Harnesk vs. Paragon } & \multicolumn{2}{c}{ Harnesk vs. Solstice } & \multicolumn{2}{c}{ Paragon vs. Solstice } \\
\cline { 2 - 6 } & Crown & Leaf & Crown & Leaf & Crown & Leaf \\
\hline 2 Fold & 1326 & 775 & 920 & 1236 & 1943 & 1734 \\
5 Fold & 268 & 186 & 168 & 318 & 299 & 437 \\
\hline
\end{tabular}

Values derived from a volcano plot of 2 and 5 fold differences with t-test $p$ values of 0.05 .

consistent with the hypothesis that TaVRN1 is a promoter of flowering induced by extended periods of cold. However, our experimental design gave us the opportunity to make an additional and interesting observation with regard the regulation of TaVRN1. That is, the TaVRN1 transcript accumulated in a similar fashion, although to a lesser extent, in both vernalised plants and controls plants exposed to a gradual decline in light intensity and day-length but not a decline in temperature (Figures 3a, b). Our results suggest that TaVRN1 expression might be influenced by both light and temperature, as has recently been reported by Hemming et al. (2008). Indeed, the two stimuli might act synergistically since there was greater accumulation of transcript in the vernalised plants, which experienced the influence of both cues, than in the control plants, which experienced only a decline in day-length. Alternatively, we cannot exclude the possibility that this important promoter of flowering gradually accumulates through time allowing plants to eventually become competent to flower even in the absence of cold. Thus, given the later hypothesis, even following a mild winter, winter varieties would eventually accumulate enough TaVrn1.p to permit flowering.

Whereas, TaVRN1 behaved in a manner consistent with it being a promoter of flowering, the profiles of abundance of the TaVRN2 transcript were not what would be expected of a repressor of flowering, and do not fit the model presented in Additional file 1 (model a). In crown tissue, where one might have expected to see a response, since the perception of cold is thought to occur in actively dividing cells of the shoot apical mersitem, transcript abundance remained low (below the level of detection with qRT-PCR) and unchanging in all three varieties. In leaves of the winter varieties, there was a decline in abundance between weeks five and nine - a 2.5-fold and 1.7fold decline, respectively, in Harnesk and Solstice. But, even at their highest point, transcript levels were very low - result confirmed by qRT-PCR (Pearson correlation = $0.95)$. The lack of correspondence between our results for TaVRN2 and those of other groups may find explanation in the different experimental designs used. Our growth conditions were designed to mimic the autumn to winter transition. In other studies, continuous long-day condition were used in combination with either constant low temperature $\left(4^{\circ} \mathrm{C}\right)$, or a one-phase shift from high to low temperature $[2,16,21]$. Regardless of the differences in growth conditions, the precise role of TaVRN2 in vernalisation has already been questioned. In a recent paper, [22] it was shown that its expression remained low when plants were vernalised under short day conditions, and suggested that TaVRN2 is probably not a repressor of TaVRN1. In addition, it has been suggested that TaVrn2.p is not able to bind the TaVRN1 promoter [12] and so can't act as a direct repressor of its expression. Our results for TaVRN2 correspond with those of Trevaskis et al. [22] then, rather than with results that indicate a direct role for VRN2 in vernalisation.

Recent discussions of the genetic control of phase transition in barley and wheat have not only brought into question the role of TaVRN2 $[23,24]$ but indicate that genes once thought to be exclusively controlled by temperature may also respond to day-length $[2,12,25,26]$. A third gene, TaVRN3, a component of the photoperiod pathway thought to be a promoter of TaVRN1 expression, is upregulated by long-days. Alternative models for the relationship between these three genes are depicted in Additional file 1 (models a and b). The third component of the models of phase transition presented in Additional File 1 is TaVRN3, which is thought to be an orthologue of AtFT (FLOWERING LOCUS T). According to Yan and co-workers [24], TaVrn3.p is a flowering promoter which is upregulated by long days. In turn, TaVrn3.p positively regulates TaVRN1. In this model, both TaVRN1 and TaVRN3 are negatively regulated by $\mathrm{TaVrn} 2$.p. Vernalisation results in a decrease in TaVrn2.p and the de-repression of TaVRN1 and TaVRN3. Significantly, it was suggested [24] that transcript levels of all three genes remain low under short day conditions, and that only on transfer to long days are TaVRN1 and TaVRN3 up-regulated and able to initiate the cascade of events that result in flowering. Our results for TaVRN3 agree with those of Yan et al. [24]: transcript levels of TaVRN3 remained low and unchanging throughout the experiment in all three varieties. That is, the plants were grown under short-day conditions, and so TaVRN3, which requires long-days, was not expressed.

This negative result is, of itself, quite interesting since it indicates that TaVrn3.p is not required for TaVRN1 to be 


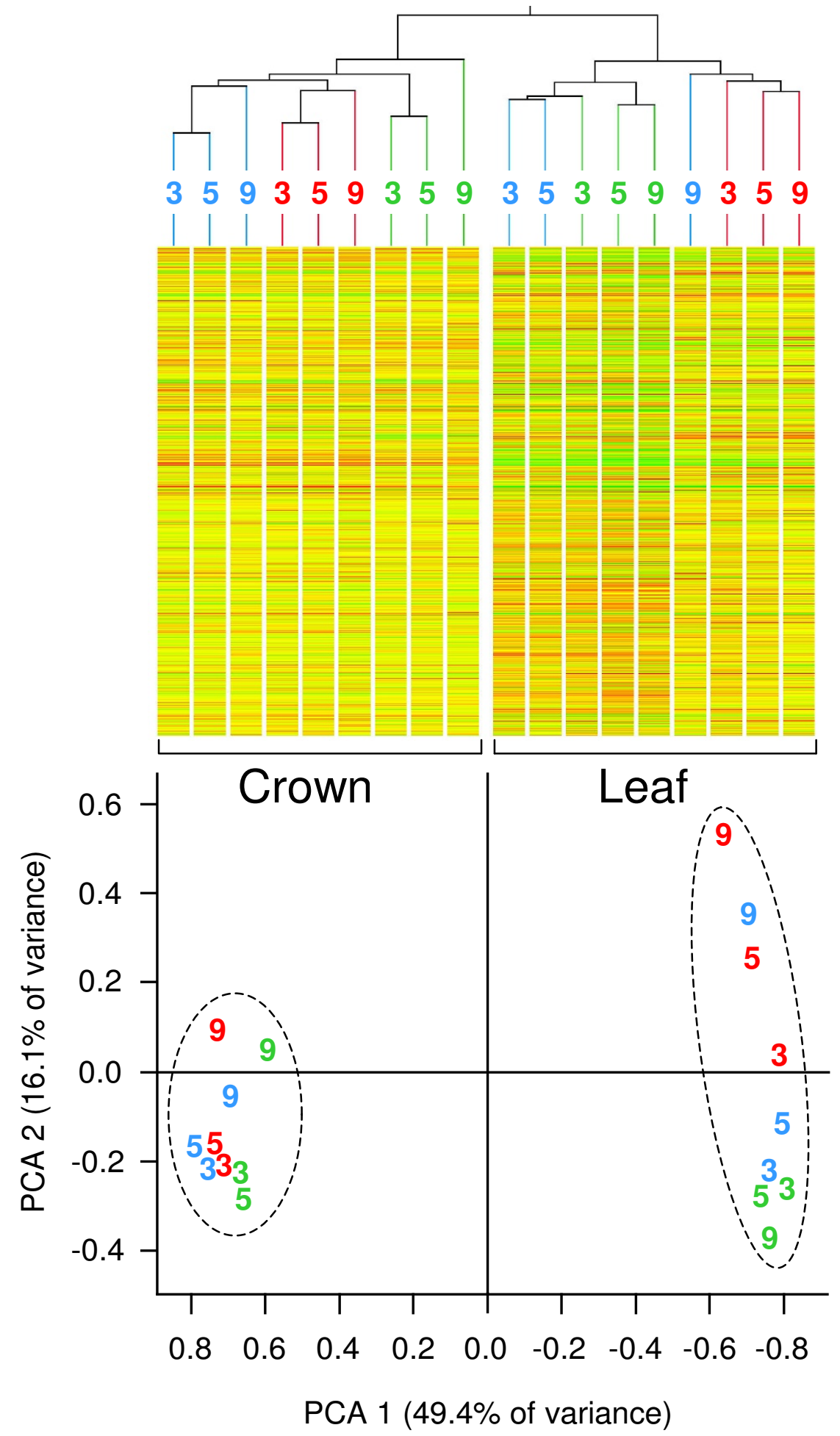

\section{Figure 2}

Condition tree and PCA plot based on gene expression profiles of the three cultivars for 3 time points: 3 weeks, 5 weeks and 9 weeks post-germination (marked on plots). In both plots, the samples of Harnesk are highlighted in blue, Solstice in red, and Paragon in green. 

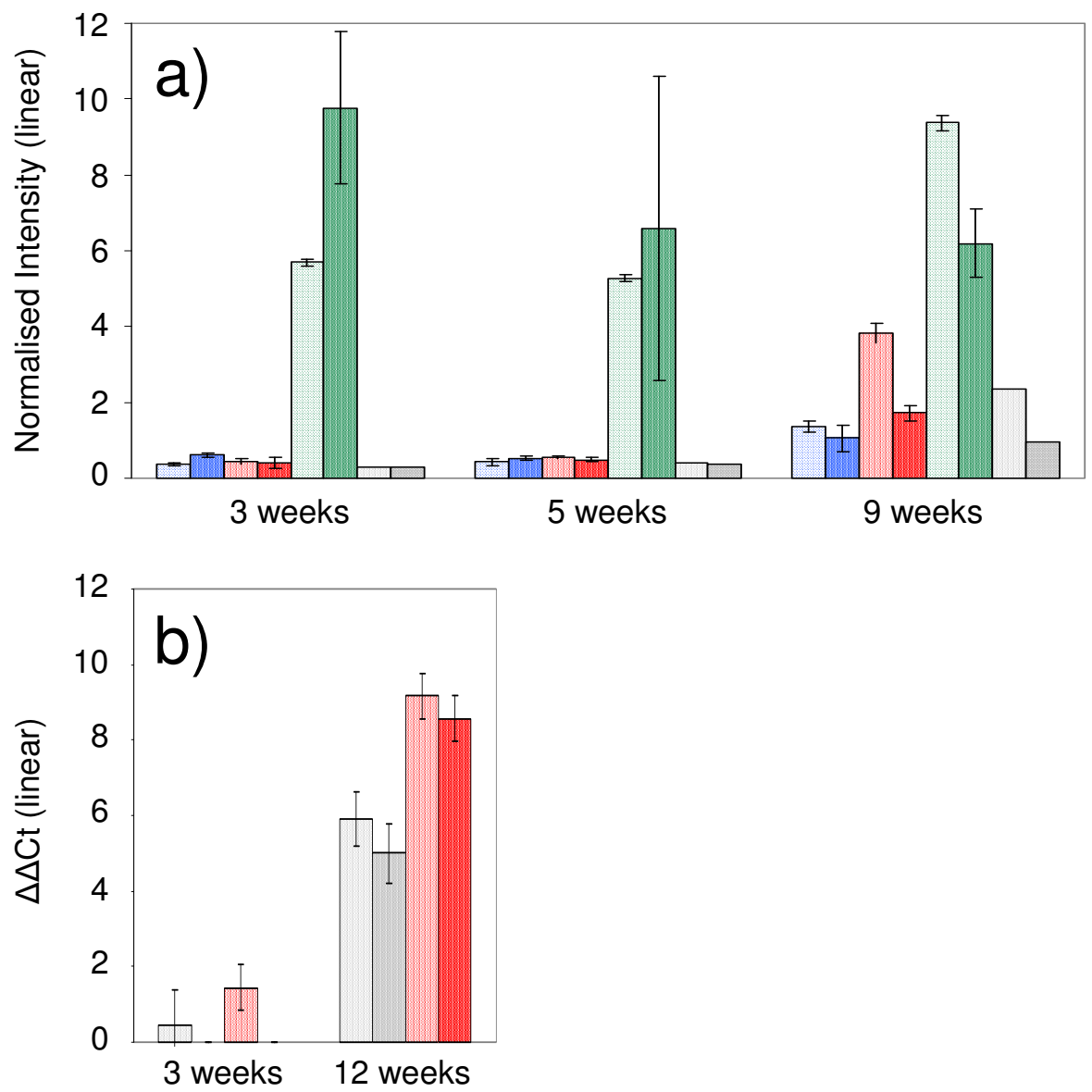

\section{Figure 3}

Bar diagram of TaVRN I transcript abundance: a) a comparison of array data of the three varieties at 3, 5 and 9 weeks; b) a comparison of qRT-PCR data of vernalised Solstice and non-vernalised controls at 3 and I 2 weeks. Colour code: Harnesk = blue; Solstice = red; Paragon = green; Control = grey; in each case the lighter shade represents crown tissue.

expressed; regardless of the low abundance of the TaVRN3 transcript, transcripts of TaVRN1 exhibited an increase over the time-course.

In the light of the evidence against TaVRN2 being a repressor of TaVRN1, the group of Trevaskis $[23,27]$ has recently proposed a model in which TaVRN2 is presented as a possible integrator of the vernalisation and photoperiod pathways. In this model, the principle relationship between TaVRN1 and TaVRN2 has been reversed with respect to earlier models; that is, TaVRN1 negatively regulates TaVRN2 (see Additional file 1, model b). In this model, TaVRN2 is up-regulated under long-days before the onset of winter and, in the absence of TaVrn1.p, represses TaVRN3. After vernalisation (after winter, therefore), even though days are lengthening, TaVrn1.p is abundant and represses TaVRN2, which, in turn, removes the repression of TaVRN3 (synonymous with TaFT1). This model might go some way to explain the two steps of phase transition: i) competence to flower as a result of the cold of winter; ii) commitment to flowering as temperatures rise and days lengthen in the spring.

\section{Wheat orthologues and functional equivalents of Arabidopsis floral pathway genes}

There are four major genetic pathways that regulate vegetative to reproductive transition in Arabidopsis: the photoperiod and vernalisation pathways which mediate responses to light and cold, respectively, and the autonomous and gibberellin pathways that are regulated by endogenous signals [7-10]. We tried to identify all the probe-sets on the Affymetrix Genechip Wheat Genome Array that correspond to orthologues, or are functional equivalents, of Arabidopsis genes involved in the four path- 
ways that control flowering (see Additional file 3). Obviously, this was not possible in all cases. However, the major components of the photoperiod pathway in Arabidopsis do have orthologues in the cereal monocots $[8,28,29]$, and two of the major component of the autonomous pathway in Arabidopsis, AtFCA and AtFY, are also conserved in monocots [28]. Using both nucleotide and protein sequences of Arabidopsis genes, BLAST searches were made to identify cereal genes with high sequence similarity. These sequences were then used to search the NETAFFX database of probe-sets on the Wheat Array http://www.affymetrix.com/analysis/index.affx. Of the 24 targets that correspond to Arabidopsis phase transition genes, nine didn't show statistically significant changes in abundance (two-fold, p 0.05), and were not considered further. The other 15 showed abundance profiles that included two-fold or greater change.across the timecourse.

\section{GA pathway genes}

The Affymetrix array doesn't include probe-sets for AtGA1 (codes for ent-copalyl diphosphate synthase) or AtGA INSENSITIVE (the wheat orthologue is REDUCED HEIGHT B1 [RHT B1]). There is a probe-set for AtRGA1 (the wheat orthologue of RHT D1), but we did not observe differential accumulation of this gene. However, there was a clear genotype-dependent, cold response of some components of the gibberellin pathway: transcripts for ent-kaurene synthase and ent-kaurene oxidase (correspond to AtGA2 and AtGA3, respectively), showed leaf specific accumulation ( $>20$-fold increase after 12 weeks) in the two winter varieties and no response at all in Paragon (Figure 4). This result was confirmed by qRT-PCR (Pearson correlation $=0.99)$. Ent-kaurene synthase and ent-kaurene oxidase are two of the principal enzymes of the gibberellin biosynthetic pathway $[30,31]$. Thus, given

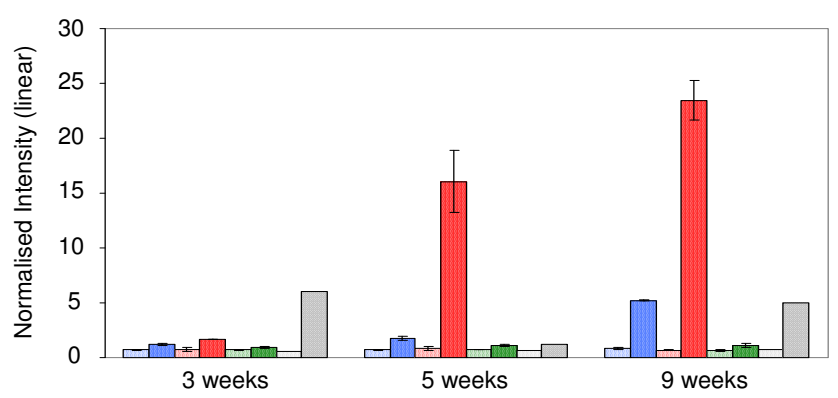

Figure 4

Bar diagram of Ent-kaurene oxidase transcript abundance: a comparison of array data (normalised intensity) of the three varieties at 3, 5 and 9 weeks. Colour code: Harnesk = blue; Solstice = red; Paragon = green; Control = grey; in each case the lighter shade represents crown tissue. the profiles of abundance that we observed, one might assume that, in the two winter varieties, there was an increase in gibberellins. In Arabidopsis, gibberellic acid (GA) activates the expression of AtSOC1(SUPPRESSOR OF OVER EXPRESSION OF CO 1) [32], an important integrator of several flowering pathways (discussed below), which in turn promotes flowering through its action on floral meristem identity genes or their products (Komeda 2004). What's more, Moon et al. [32] report that the gibberellin pathway is the only pathway to promote flowering under short days. Thus, it would appear that we have evidence to show that in wheat the gibberellin pathway functions in a similar manner to that in Arabidopsis, and that as a consequence of vernalisation under short-days it tends to promote flowering. However, the complete lack of response in the spring variety, Paragon, is intriguing: does the gibberellin pathway not function to promote flowering in spring varieties of wheat?

\section{Autonomous pathway}

In Arabidopsis, seven genes (AtFCA, AtFY, AtFLD, AtFVE, AtFPA, AtLD and AtFRI) have been shown to comprise the autonomous pathway $[7,8]$. All of these, except AtFRI, are thought to promote flowering by repressing AtFLC, a dominant repressor of flowering. AtFRI, on the other hand, upregulates expression of AtFLC and so represses flowering. No orthologues for AtFRI or AtFLC have been found in the cereals (Alexandre and Hennig, 2008). Orthologues (or, at least, genes which code for proteins with similar structure and function) of the other six are present in the cereals [28], but there are probe-sets for only two of these (TaFVE and TaFCA) on the wheat array (see Additional file 3). Both these genes showed a slight increase in abundance across the time-course. However, there were no particular differences in response between the winter and spring varieties. In addition, in vernalised and control plants transcripts for the two genes exhibited similar profiles of abundance, as might be expected for genes that are not thought to be responsive to light or cold [8].

\section{Photoperiod pathway}

The principal components of the photoperiod pathway are conserved in the monocots and, more pertinent to this discussion, in the cereals $[8,33,34]$. On the Wheat Genome Array, there are probe-sets that correspond to many of the genes belonging to the photoperiod pathway (Additional file 3).

In Arabidopsis, AtCONSTANS (AtCO) encodes a transcription factor that activates genes required for floral initiation. It integrates circadian clock and day-length signals and, under long-days, activates the floral promoters AtFT, AtSOC1 and AtLFY [8]. The two circadian clock genes $A t L H Y$ and AtTOC1 influence the expression of AtCO. 
They form part of a feedback mechanism, each directly affecting the expression of the other: AtLhy.p is a repressor of AtTOC1, and AtToc1.p is required for the expression of AtLHY [8]. The cyclic expression of these two genes, which occurs over a 24 hour period, entrains that of AtGIGANTEA (AtGI). This latter activates AtCONSTANS.

In this study, the profiles of abundance for TaLHY and TaTOC1 were complementary to each other, as one might expect from their relationship to each other in circadian cycling. In crown tissue, transcript of TaLHY increased in abundance and then declined; conversely, that of TaTOC1 declined and later increased. The profile for $\mathrm{TaGI}$ was very similar to that of TaTOC1 (see Additional file 4). Given that in both rice and Arabidopsis, GI is a promoter of $\mathrm{CO}$ expression [8], one might have expected the transcript for HEADING DATE 1 (TaHD1), the supposed wheat orthologue of AtCONSTANS, to follow the profile of TaGI. However, it did not show differential expression in this study. Interestingly, other transcripts that appear to be members of the CONSTANS-like family of genes exhibited profiles that did reflect those of TaLHY, and TaTOC1, and TaGI. In particular, a sequence highly similar to barley CONSTANS-like 9 (the most divergent of the barley CONSTANS-like genes which has no counterpart in Arabidopsis [35]) had a profile of abundance very similar to that of TaLHY (see Additional file 4). A transcript with similarity to CONSTANS-like 1 in Lolium perenne, a gene which has been reported to increase after extended periods of exposure to cold [36], had a profile of transcript abundance that echoed that of TaTOC1 and TaGI. Ciannamea et al., using a similar experimental approach to that used in this study, suggested that the profile of transcript abundance for LpCOL1 was suggestive of the gene being involved in the vernalisation response [36]. We observed a very similar profile of responses in both the cold treated plants and the controls. This would suggest that this gene in wheat is responding to shortening day length.

The phytochromes (perceive red and far-red light) and the cryptochromes (perceive blue and UV-A light) are the principle components involved in the perception of light. What is more, phytochromes regulate a variety of developmental processes, and are thought to be involved in signaling, probably through the possession of a kinase domain residing within their C-terminal domain [13]. Within the photoperiod pathway, it is believed that, under long-day conditions, these photoreceptors contribute to the initiation of flowering through the stabilisation of the $\mathrm{CO}$ protein [37]. Of the principal photoreceptors, only three, TaCRY2, TaPHYA and TaPHYC, were identified on the Affymetrix Wheat Genome Array. Two of these, TaCRY2 and TaPHYA, exhibited a response under our experimental conditions. Transcript of TaCRY2 accumulated, principally in leaf tissue, of the two winter varieties under both the vernalisation regime and in the control plants. In Paragon, no statistically significant change in transcript abundance was observed. Transcript levels of TaPHYA were initially much higher in the crown tissue of the two winter varieties than in Paragon and increased across the time course. There was also an increase in transcript abundance in leaf tissue of the winter varieties, but from a lower initial level. The same pattern of increase was seen in the controls.

\section{Intergrative pathway}

In Arabidopsis, the four floral pathways converge through genes of the integrative pathway [7]. The activation of floral integrators, such as AtFLOWERING LOCUS T (AtFT) and AtSUPPRESSOR OF CONSTANS 1 (AtSOC1), in turn, lead to the activation of floral meristem identity genes such as LEAFY (LFY) and APETALA1 (AP1). We did not observe differential expression of TaFT (see above discussion of TaVRN3). On the other hand, transcripts for wheat genes that share sequence similarity with AtSOC1 did show differential expression. Zhao et al. [38] identified seven MADS-box genes (TaAGL1, TaAGL7, TaAGL18, TaAGL20, TaAGL21, TaAGL23 and TaAGL38) that, upon phylogenetic analysis, were placed in the SOC1-like clade of MADS-box genes. Only three probe-sets on the wheat array corresponded with these seven genes (see Additional file 5). The gene TaAGL7 corresponds with the probe set Ta.25343. The genes TaAGL1, TaAGL18 and TaAGL23 (the most similar to AtSOC1) all correspond to one probe-set (Ta.21250), and TaAGL20, TaAGL21 and TaAGL38 (the least similar to AtSOC1) to another (TaAffx.19661) given the high sequence similarity between the genes in the respective groups, they are probably homoeologues and so we cannot report on their individual behaviour. However, the three probe-sets that correspond to the genes of the SOC1 clade of MADS-box genes all evidenced essentially the same profile of abundance (data not shown). That is, in both leaf and crown tissue of all three varieties, transcript increased slightly (Figure 5).

In Arabidopsis, AtSOC1 acts as a integrator of several floral induction pathways, and is induced by vernalisation [39]. Genes from both rice (OsMADS50) and ryegrass (LpMADS1, LpMADS2, LpMADS3) that share sequence similarity with AtSOC1, and may be functional equivalents of it, are also reported to accumulate as a consequence of vernalisation $[40,41]$. We observed a slight increase in abundance in wheat, but the profile of abundance in the vernalised and control plants were essentially identical. Thus, we might not be observing a response to declining temperature but to declining day-length. Alternatively, as indicated by Shitsukawa et al. [42], it might well be that this gene is neither influenced by vernalisation nor day-length. Indeed, this latter group present a 


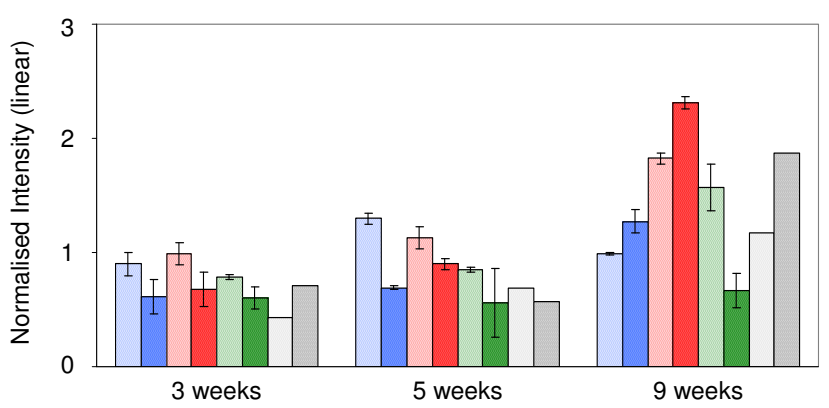

Figure 5

Bar diagrams of transcript abundance of the MADSbox gene TaAGL2I, the gene most similar to AtSOCI: a comparison of array data (normalised intensity) of the three varieties at 3, 5 and 9 weeks post-germination. Colour code: Harnesk = blue; Solstice $=$ red; Paragon $=$ green; Control = grey; in each case the lighter shade represents crown tissue.

model in which WSOC1 acts as an activator of flowering that is influenced by the Gibberellin pathway [42].

\section{Other MADS-box genes}

Members of the MADS-box gene family encode transcription factors that play a fundamental role in signal transduction and control of development in probably all eukaryotes [43]. For instance, floral organ identity genes (homeotic genes) of the ABCDE model of floral organ development are mostly MADS genes [44]. In rice and Arabidopsis, species for which sequencing has been completed, 73 and 107 MADS-box genes have been identified, respectively [38]. In wheat about 50 MADS-box genes have been identified; these are dispersed throughout the genome $[38,45]$. We identified all the features on the wheat array that correspond with MADS-box genes (see Additional file 5) to determine whether they exhibited abundance profiles indicative of involvement in phase transition.

The MADS-box gene VEGETATIVE TO REPRODUCTIVE TRANSITION 2 (TaVRT2), the product of which shares $51 \%$ sequence identity with the Arabidopsis protein SHORT VEGETATIVE PHASE (AtSvp.p), has been reported to play an important role in phase transition in wheat $[12,46]$. Kane et al. [46] reported that, in spring wheat, levels of TaVrt2.p remain low and stable under cold treatment, whilst in winter wheat it starts higher than in spring wheat and then declines. This pattern of expression, reminiscent of that reported for TaVRN2, is consistent with the hypothesis that TaVRT2 is a repressor of flowering. Indeed, Kane et al. [12] suggested that TaVrt2.p (as part of a hypothesised protein complex, possibly with TaVrn2.p) might bind to the promoter region of TaVRN1 and repress it. However, Trevaskis et al. [12,26], working with barley, suggested that this is unlikely to be the case: they failed to find any evidence for there being a direct interaction between $H v V R T 2$ and HvVRN1 (the barley homologues of TaVRT2 and TaVRN1, respectively) and showed that $H v V R T 2$ transcript abundance increased during cold treatment. In our study, in both tissues of all three varieties, there was an increase in transcript abundance (approx. 1.5 to 2.0 fold) as temperature decreased. Thus, our results are in agreement with those of Trevaskis et al. [26]. If indeed VRT2 is a repressor of flowering, the profiles observed here could be explained by assuming that, in both winter and spring varieties, there is a tendency to retard flowering until permissive warm, long-day conditions return. That is, TaVRT2 might not repress TaVRN1 but counteract its function and so be part of a mechanism to hold back flowering until the return of spring. Indeed, it has been reported that short days repress reproductive development in spring varieties [47]. That is, plants that have no vernalisation requirement use day-length as a cue to retard flowering until the permissive temperatures and lengthening days of spring stimulate them to flower. Thus, TaVRT2, a repressor of flowering, and TaVRN3, a floral promoter, might work in concert as part of a mechanism to check the flowering in vernalisation saturated plants (or, indeed, in plants that don't require cold to acquire competence to flower) until day-length is appropriate.

Finally, several less well studied MADS-box genes behaved in an intriguing manner suggestive of their involvement in vernalisation or photoperiod induced phase transition. Of particular interest were TaAGL10, TaAGL33 and TaAGL42. The gene TaAGL10 belongs to the same subfamily of MADS-box genes as TaVRN1 although their proteins shares only $52 \%$ identity [38]. Interestingly their profiles of expression in crown tissue were quite similar there was little response of TaAGL10 in leaf tissue of any of the three varieties. That is, at all three time points, transcript in Paragon was much higher than in the two winter varieties. In the latter, transcript was initially very low but began to accumulate by the ninth week. Analysis with qRT-PCR showed that this increase continued to the twelfth week (data not shown). The similarity between the profiles of TaVRN1 and TaAGL10 suggests that they might respond to the same cues. Further work will need to be carried out to determine the downstream interaction of the TaAgl10 protein.

The two genes TaAGL33 and TaAGL42 are Class I MADSbox genes. The precise function of genes belonging to this class is not well understood $[48,49]$. However, both TaAGL33 and TaAGL42 are closely related to the rice gene OsMADS51[38] which, in rice, has been shown to be a flowering activator under short-days[50]. In our experiment, differential expression of these two genes was restricted to the two winter varieties (Figure 6). At three 

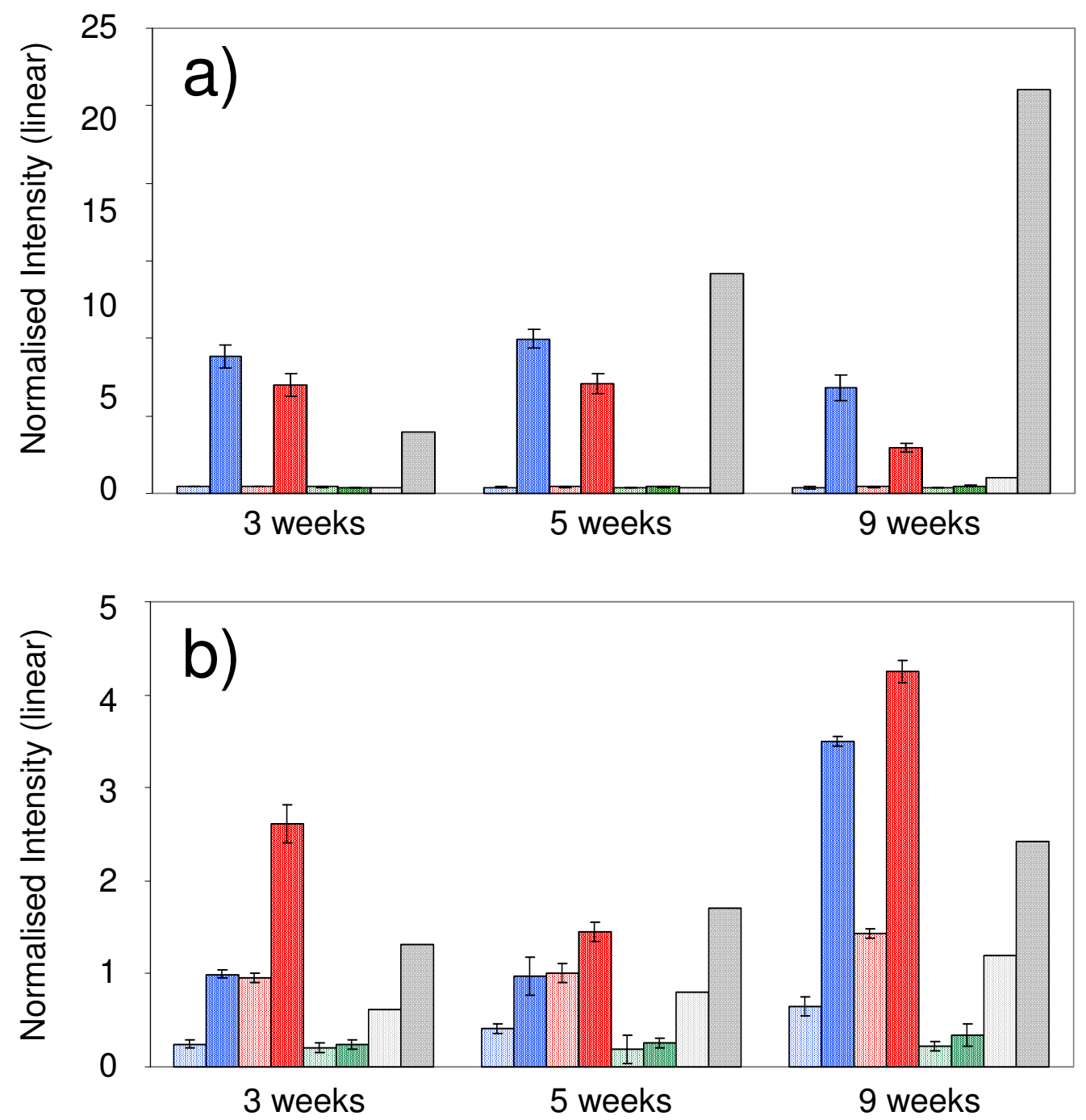

Figure 6

Bar diagrams of transcript abundance of a) TaAGL33 and b) TaAGL42: a comparison of array data (normalised intensity) of the three varieties at 3, 5 and 9 weeks post-germination. Colour code: Harnesk = blue; Solstice = red; Paragon = green; Control = grey; in each case the lighter shade represents crown tissue.

weeks, transcript of TaAGL33 was relatively high in leaf tissue of the two winter varieties, but declined between the fifth and ninth weeks. This pattern corresponds with the results reported by Trevaskis et al., [21] for the Type I MADS-box gene, TaMx23. A BLAST search using the sequence of TaMX23 (accession number BJ258117) shows that the two sequences are very similar (85\% identify). However, TaM $x 23$ is more similar to the sequence of TaAGL42 (95\% identity) which showed a different profile of abundance. Transcript of TaAGL42 increased, principally in leaf tissue, in the winter varieties and showed no response in Paragon. If these two MADS-box genes are involved in cold induced phase transition, their profiles of abundance would suggests that TaAGL33 is a repressor of flowering and TaAGL42 a promoter. The complete lack on response of both genes in the spring wheat, Paragon, is quite intriguing; it would indicate that in spring varieties these genes are constitutively repressed or that they occur as non-functional alleles. This would make sense for the TaAGL33 transcript assuming that it were a repressor of flowering. That is, in winter varieties it declines as a consequence of vernalisation, whilst in spring varieties it always remains low or absent. However, the role that TaAGL42 might have in phase change is more difficult to interpret. If one were to hypothesise that TaAGL42 generally acts to promote flowering then it should be constitu- 
tively expressed in spring varieties: it remained low and unchanging in both tissues, however. Thus, TaAGL42 might not be involved in phase transition at all. A clue that instead it might be involved in cold acclimation is given by the fact that, of the MADS-box genes on the array, it is the only one apart from TaVRT-2 that responded to a cold "shock". That is, in a separate experiment (not described here) in which plant were exposed to a rapid drop in temperature from $15^{\circ} \mathrm{C}$ to $4{ }^{\circ} \mathrm{C}$ and held for two days, transcript of TaAGL42 in the two winter wheat varieties increased in abundance four- to five-fold over a two day period. Transcript for TaVRT-2 approximately double in all three varieties over the same period whilst transcript for all other MADS-box genes studied showed no statistically significant change in abundance (Figure 7).

\section{Conclusion}

This study, we believe, is novel in that it was carried-out under conditions that closely reflect those that typically occur during the winter in a northern climate - a gradual decline in temperature, day-length and light intensity. In most previous studies, unnaturally high light intensities have been maintained throughout the experiment, or sudden drops in temperature have been used. These conditions may have given rise to unusual, stress-related responses. Certainly, we have observed expression patterns for some genes that call into question earlier studies.

In choosing a microarray approach we have obtained a broad overview of the behaviour of the wheat transcriptome to winter conditions and this has provided many interesting clues to the interaction of the several pathways involved in phase transition. We have also seen how these might differ between different tissue and, particularly, between winter and spring varieties of wheat. With regard to many genes, we observed patterns of transcript accumulation that reflect those seen by other groups in other studies. However, as a consequence of the conditions under which we grew our plants, we have also made observations that give additional insight to the complexities of the interaction that occur between floral pathways, and how internal and external cues come into play to ensure flowering occurs when internal and external cues are appropriate. For instance, it has not been previously reported that TaVRN1 might respond to light - thus, it might be that TaVRN1 is involved in pathways other than just the vernalisation pathway. The lack of a clear response of TaVRN2 was also unexpected when we began our study, but has recently been discussed by other groups [22]. Our results support the view that this gene is probably not directly involved in vernalisation as was, until recently, thought to be the case. Clear evidence that the Gibberellin pathway is important in wheat and, by extension, in other cereals too, is also interesting. Finally, we have highlighted genes that certainly warrant further

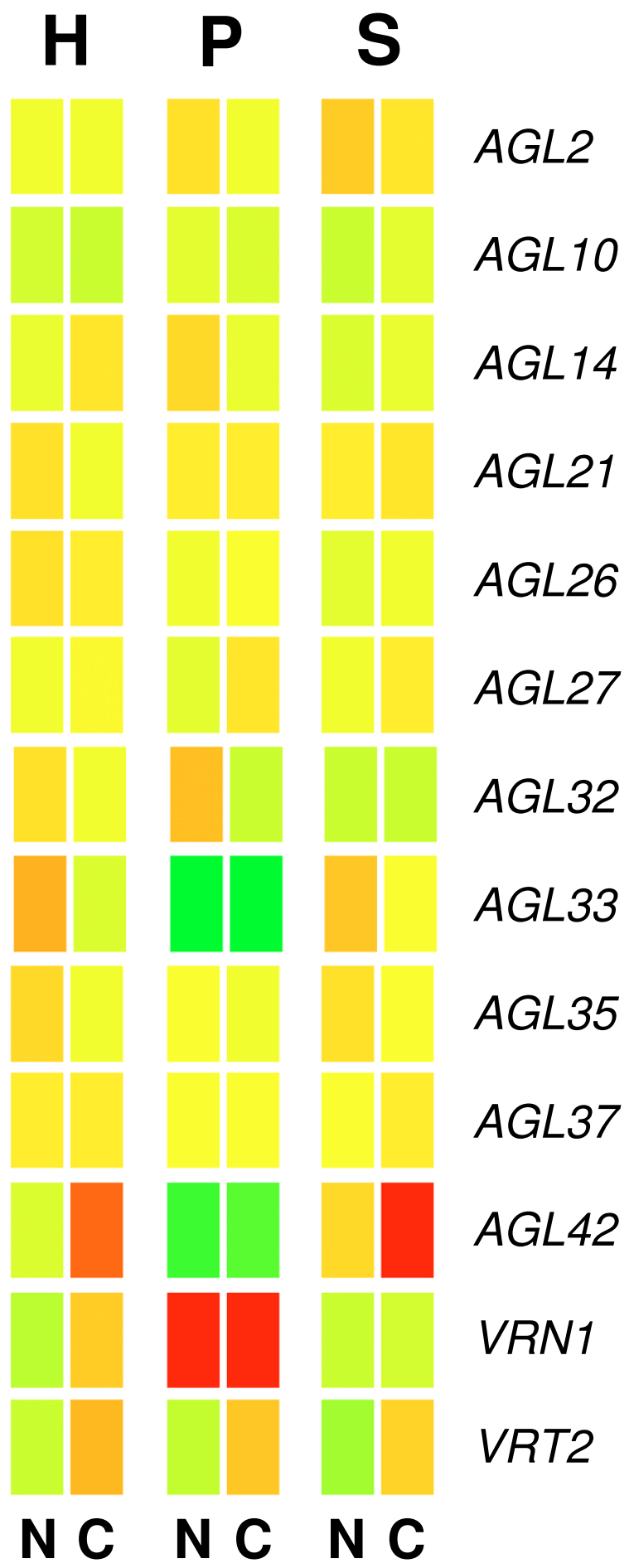

Figure 7 


\section{Figure 7}

Relative expression of MADS-box genes in plants exposed to a sudden drop in temperature from $15^{\circ} \mathrm{C}$ to $4^{\circ} \mathrm{C}$. Most MADS-box genes didn't show a statistically significant response to the cold shock. In the two winter varieties, TaAGL42 showed a marked increase in expression in the cold treated plants relative to the controls $(>3$-fold change, $p=0.05)$.TaVRT2 showed a $>1.5$-fold increase in all three varieties $(p=0.05)$. Code: $H=$ Harnesk; $P=$ Paragon; $S=$ Solstice; $N=$ no cold treatment; $C=$ cold shock.

investigation such as the MADS-box genes TaAGL10, TaAGL33 and TaAGL 42.

On a broad scale, it seems evident that phase transition and flowering in cereals, as in Arabidopsis, is controlled by several different pathways that allow plants to respond to different external (temperature, day length and light quality) and internal (gibberellin and autonomous pathways) cues. The different pathways, each responding to their own cues and each promoting or repressing flowering depending on the message provided by these cues, provide the checks and bounds to flowering. Thus, only when plants are sufficiently mature and receive the appropriate cues of temperature, day-length and a light quality will they proceed to flower. A greater knowledge of the precise details of these pathways will allow for the selection or breeding of varieties to match particular environments.

\section{Methods \\ Plant material}

Three photoperiod responsive wheat cultivars were used for the project: Harnesk and Solstice, both winter varieties, and Paragon, a spring variety. Seeds were planted in 50:50 potting compost:perlite in $10 \mathrm{~cm}$ pots and maintained in growth cabinets with a $14 \mathrm{~h}$ photoperiod (280) and $16^{\circ} \mathrm{C}$ day $/ 14^{\circ} \mathrm{C}$ night temperature. Three weeks postgermination, experimental procedures began (Table 1). As a control, plants of Solstice were grown under identical conditions of light and day-length as the experimental plants but without a corresponding fall in temperature.

Samples were taken for analysis on the last day of each time period/temperature segment: 3, 5, 7, 9 and 12 weeks. Harvesting took place in the middle of the photoperiod to avoid, as much as possible, the influence of circadian rhythms.

\section{RNA extraction and purification}

Total RNA was independently prepared from two separate tissues: crown and leaf. Because of problems of handling, "crown tissue" constituted the apex and some subtending stem including the vascular transition zone and immature leaf bases. In each case, RNA was extracted from material pooled from 4 separate plants. Tissues from control plants were harvested at the same time as the experimental plants. The samples for RNA extraction were frozen in liquid nitrogen, and stored at $-80^{\circ} \mathrm{C}$ until used.

RNA was extracted using the TRIZOL (Invitrogen) method reported in [51].

\section{Photographic imaging}

Crown tissues were collected from plants and fixed and embedded in Lambwax (Raymond A. Lamb Ltd., Eastbourne, BN23 6QE, UK) using standard histochemical techniques [52]. De-parafinised sections were re-hydrated and subsequently stained with $0.05 \%(\mathrm{w} / \mathrm{v})$ toluidine blue in $10 \mathrm{mM}$ sodium benzoate $\mathrm{pH} 4.4$. Slides were examined at low magnification under bright-field illumination using a Wild Photomacroscope IV (Leica Microsystems (UK) Ltd.). A Leica DMRB microscope (Leica Microsystems (UK) Ltd.) was used for high magnification observations and for the capture of photographic images.

\section{Array analysis}

Affymetrix Genechip Wheat Arrays (Affymetrix, USA) were used. Hybridisation, washing and staining were performed according to manufacturer's instructions. Microarray data were extracted from scanned GeneChip images and analyzed using Microarray Suite version 5.0.1 (Affymetrix). Array normalisation and analysis were performed using the GeneSpring GX 7.3 software package (Agilent): the raw data, imported as CEL files, were normalised per chip using RMA (log-scale Robust Multi-array Analysis) open access GeneSpring software; per gene normalisation to the median was performed within the programme. These data are available for public access: the GEO accession number for array data is GSE11774.

\section{Real-time quantitative RT-PCR}

To confirm expression patterns observed from the analysis of array data, real time qRT-PCR experiments were performed using an ABI Prism 7000 SDS (Applied Biosystems, U.K.). Reverse transcription was performed using Superscript III First Strand Synthesis Kits (Invitrogen) according to manufacturer's instructions. Quantitative RTPCR was performed using SYBR Greener qPCR Supermix for ABI Prism (Invitrogen). The primers used are available in Additional file 6 . Reaction conditions were as follows: $50^{\circ} \mathrm{C}$ for 2 mins; $95^{\circ} \mathrm{C}$ for 10 mins; 42 cycles of $95^{\circ} \mathrm{C}$ for $15 \mathrm{~s}$ and $58^{\circ} \mathrm{C} 1 \mathrm{~min}$. A dissociation protocol was performed in all cases. Quantification for each primer pair and cDNA template combination was performed in triplicate for each of the two biological replicates at every time point. 
Relative quantification was performed using the $\Delta \mathrm{Ct} \Delta \mathrm{Ct}$ method with wheat ACTIN used as the endogenous control. Correlation between results from the array data and qRT-PCR was tested using Pearson Correlation Coefficient.

\section{Authors' contributions}

MW carried some of the microarray work, and all of the qRT PCR work. He also performed all of the data analysis and wrote the manuscript. CL grew the plants, collected all tissues for analysis. He performed all RNA extractions and prepared samples for array analysis. JAC performed quality control of RNA and carried out the array analysis. IDW and KJE designed the experimental programme, and gave technical and intellectual guidance.

\section{Additional material}

\section{Additional file 1}

Proposed models for the interaction between the three genes TaVRN1, TaVRN2 and TaVRN3. Models of the interaction between the three genes TaVRN1, TaVRN2 and TaVRN3 (syn. with TaFT) redrawn from those proposed by: a) Yan et al., 2006; b) Trevaskis et al., 2007. Click here for file

[http://www.biomedcentral.com/content/supplementary/14712229-9-55-S1.doc]

\section{Additional file 2}

Vernalised and non-vernalised plants of the wheat varieties Harnesk, Solstice and Paragon. A) Photographs of non-vernalised plants of varieties Harnesk, Solstice and Paragon 5 months post-germination. B) Photographs of vernalised plants of Harnesk, Solstice and Paragon 5 months post-germination.

Click here for file

[http://www.biomedcentral.com/content/supplementary/14712229-9-55-S2.doc]

\section{Additional file 3}

Genes potentially involved in phase transition. A table listing the features on the Affymetrix Genechip Wheat Genome Array that are reported to be homologues of, or are highly similar to, genes involved in phase transition in Arabidopsis.

Click here for file

[http://www.biomedcentral.com/content/supplementary/14712229-9-55-S3.doc]

\section{Additional file 4}

Profiles of transcript abundance for photoperiod pathway genes discussed in article. Profiles of transcript abundance for the photoperiod pathway genes. Only those transcripts that showed a statistically significant 2-fold or greater change in abundance are represented. a) PHYA, b) TOC1, c) LHY, d) GIGANTEA, e) COL1 and f) COL9.

Click here for file

[http://www.biomedcentral.com/content/supplementary/14712229-9-55-S4.doc]

\section{Additional file 5}

List of the 42 wheat MADS-box genes described in Zhou et al., 2006. A list of the features on the Affymetrix Genechip Wheat Genome Array that correspond to the wheat MADS-box genes described in the article by Zhou et al., 2006 [reference [38]].

Click here for file

[http://www.biomedcentral.com/content/supplementary/14712229-9-55-S5.doc]

\section{Additional file 6}

Primers for $q R T$-PCR. A table containing the details of the primer pairs used in $9 R T-P C R$.

Click here for file

[http://www.biomedcentral.com/content/supplementary/14712229-9-55-S6.doc]

\section{Acknowledgements}

We thank Steve Martin for his help on the cell imaging and Andrew Hughes for his assistance with the growing and harvesting of samples. This work was supported by Biotechnology and Biological Sciences Research Council, UK (BBSRC) Agri-Food (grant ref. BBSB02। I8).

\section{References}

I. Sung S, Amasino RM: Molecular genetic studies of the memory of winter. Journal of Experimental Botany 2006, 57:3369-3377.

2. Dubcovsky J, Loukoianov A, Fu DL, Valarik M, Sanchez A, Yan LL: Effect of photoperiod on the regulation of wheat vernalization genes VRNI and VRN2. Plant Molecular Biology 2006, 60:469-480.

3. Snape JW, Sarma R, Quarrie SA, Fish L, Galiba G, Sutka J: Mapping genes for flowering time and frost tolerance in cereals using precise genetic stocks. Euphytica 200 I, | 20:309-3 I5.

4. Sheldon CC, Finnegan EJ, Rouse DT, Tadege M, Bagnall DJ, Helliwell CA, Peacock W], Dennis ES: The control of flowering by vernalization. Current Opinion in Plant Biology 2000, 3:4I 8-422.

5. Simpson GG, Dean C: Flowering - Arabidopsis, the rosetta stone of flowering time? Science 2002, 296:285-289.

6. Amasino RM: Vernalization and flowering time. Current Opinion in Biotechnology 2005, 16:154-158.

7. Komeda Y: Genetic regulation of time to flower in Arabidopsis thaliana. Annual Review of Plant Biology 2004, 55:521-535.

8. Putterill J, Laurie R, Macknight R: It's time to flower: the genetic control of flowering time. Bioessays 2004, 26:363-373.

9. Wigge PA, Kim MC, Jaeger KE, Busch W, Schmid M, Lohmann JU, Weigel D: Integration of spatial and temporal information during floral induction in Arabidopsis. Science 2005, 309:1056-1059.

10. Jaeger KE, Graf A, Wigge PA: The control of flowering in time and space. Journal of Experimental Botany 2006, 57:34I5-34I8.

II. Lee JH, Cho YS, Yoon HS, Suh MC, Moon JH, Lee I, Weigel D, Yun $\mathrm{CH}$, Kim JK: Conservation and divergence of FCA function between Arabidopsis and rice. Plant Molecular Biology 2005, 58:823-838

12. Kane NA, Agharbaoui Z, Diallo AO, Adam H, Tominaga $Y$, Ouellet $F$, Sarhan F: TaVRT2 represses transcription of the wheat vernalization gene TaVRNI. The Plant Journal 2007, 5 I:670-680.

13. Wang H, Deng XW: Phytochrome Signaling Mechanism. In The Arabidopsis Book Edited by: Somerville CR, Meyerowitz EM. Rockville, MD: American Society of Plant Biologists; 2002.

14. Dubcovsky J, Lijavetzky D, Appendino L, Tranquilli G: Comparative RFLP mapping of Triticum monococcum genes controlling vernalization requirement. Theoretical and Applied Genetics 1998, 97:968-975.

15. Yan L, Loukoianov A, Tranquilli G, Helguera M, Fahima T, Dubcovsky J: Positional cloning of the wheat vernalization gene VRNI. Proceedings of the National Academy of Sciences of the United States of America 2003, 100:6263-6268. 
16. Yan LL, Loukoianov A, Blechl A, Tranquilli G, Ramakrishna W, SanMiguel P, Bennetzen JL, Echenique V, Dubcovsky J: The wheat VRN2 gene is a flowering repressor down-regulated by vernalization. Science 2004, 303:1640-1644.

17. Fu DL, Szucs P, Yan LL, Helguera M, Skinner JS, von Zitzewitz J, Hayes PM, Dubcovsky J: Large deletions within the first intron in VRN-I are associated with spring growth habit in barley and wheat. Molecular Genetics and Genomics 2005, 273:54-65.

18. Ndong C, Danyluk J, Huner NPA, Sarhan F: Survey of gene expression in winter rye during changes in growth temperature, irradiance or excitation pressure. Plant Molecular Biology 200I, 45:69l-703.

19. Gardner JS, Hess WM, Trione EJ: Development of the Young Wheat Spike - a Sem Study of Chinese Spring Wheat. American Journal of Botany 1985, 72:548-559.

20. Danyluk J, Kane NA, Breton G, Limin AE, Fowler DB, Sarhan F: TaVRT-I, a putative transcription factor associated with vegetative to reproductive transition in cereals. Plant Physiology 2003, I32:1849-1860.

21. Trevaskis B, Bagnall DJ, Ellis MH, Peacock WJ, Dennis ES: MADS box genes control vernalization-induced flowering in cereals. Proceedings of the National Academy of Sciences of the United States of America 2003, 100: 13099-13104.

22. Trevaskis B, Hemming MN, Peacock WJ, Dennis ES: HvVRN2 responds to daylength, whereas HvVRN I is regulated by vernalization and developmental status. Plant Physiology 2006, I 40: 1397-I 405 .

23. Trevaskis B, Hemming MN, Dennis ES, Peacock WJ: The molecular basis of vernalisation-induced flowering in cereals. Trends in Plant Science 2007, I 2:352-357.

24. Yan L, Fu D, Li C, Blechl A, Tranquilli G, Bonafede M, Sanchez A, Valarik M, Yasuda S, Dubcovsky J: The wheat and barley vernalization gene VRN3 is an orthologue of FT. Proceedings of the National Academy of Sciences of the United States of America 2006 , 103:1958I-19586.

25. Szucs P, Karsai I, von Zitzewitz J, Meszaros K, Cooper LL, Gu YQ, Chen TH, Hayes PM, Skinner JS: Positional relationships between photoperiod response $Q T L$ and photoreceptor and vernalization genes in barley. Theor Appl Genet. 2006 I I 2(7): I277-1285.

26. Trevaskis B, Tadege M, Hemming MN, Peacock WJ, Dennis ES, Sheldon C: Short Vegetative Phase-like MADS-box genes inhibit floral meristem identity in barley. Plant Physiology 2007, I 43:225-235.

27. Hemming MN, Peacock WJ, Dennis ES, Trevaskis B: Low temperature and daylength cues are integrated to regulate FLOWERING LOCUS T in barley. Plant Physiology 2008, I47(I):355-366.

28. Winichayakul S, Beswick NL, Dean C, Macknight RC: Components of the Arabidopsis autonomous floral promotion pathway, FCA and FY, are conserved in monocots. Functional Plant Biology 2005, 32:345-355.

29. Nemoto Y, Kisaka M, Fuse T, Yano M, Ogihara Y: Characterization and functional analysis of three wheat genes with homology to the CONSTANS flowering time gene in transgenic rice. The Plant Journal 2003, 36:82-93.

30. Swain SM, Singh DP, Helliwell CA, Poole AT: Plants with increased expression of ent-kaurene oxidase are resistant to chemical inhibitors of this gibberellin biosynthesis enzyme. Plant Cell Physiol. 2005, 46(2):284-29I.

31. Davidson SE, Swain SM, Reid JB: Regulation of the early GA biosynthesis pathway in pea. Planta 2005, 222:1010-1019.

32. Moon J, Suh SS, Lee H, Choi KR, Hong CB, Paek NC, Kim SG, Lee I: The SOCI MADS-box gene integrates vernalization and gibberellin signals for flowering in Arabidopsis. The Plant Journal 2003, 35:613-623.

33. Izawa T: Comparative molecular biology in photoperiodic flowering between the short-day plant rice and the long-day plant Arabidopsis. In Light Sensing in Plants Edited by: Wada M, Shimazaki K, lino M. Springer, Japan; 2005:333-337.

34. Izawa T, Takahashi Y, Yano M: Comparative biology comes into bloom:genomic and genetic comparison of flowering pathways in rice and Arabidopsis. Current Opinion in Plant Biology 2003, 6:113-120.

35. Griffiths S, Dunford RP, Coupland G, Laurie DA: The Evolution of CONSTANS-Like Gene Families in Braley, Rice, and Arabidopsis. Plant Physiology 2003, I3 I:1855-1867.
36. Ciannamea S, Busscher-Lange J, de Folter S, Angenent GC, Immink $\mathrm{RGH}$ : Characterization of the vernalisation response in Lolium perenne by a cDNA microarray response. Plant Cell Physiology 2006, 47:48I-492.

37. Valverde F, Mouradov A, Soppe W, Ravenscroft D, Samach A, Coupland G: Photoreceptor regulation of CONSTANS protein in photoperiodic flowering. Science 2004, 303:1003-1006.

38. Zhao T, Ni ZF, Dai Y, Yao YY, Nie XL, Sun QX: Characterization and expression of 42 MADS-box genes in wheat (Triticum aestivum L.). Mol Genet Genomics. 2006, 276(4):334-350

39. Lee H, Suh SS, Park E, Cho E, Ahn JH, Kim SG, Lee JS, Kwon YM, Lee I: The AGAMOUS-LIKE 20 MADS domain protein integrates floral inductive pathways in Arabidopsis. Genes \& Development 2000, I 4:2366-2376.

40. Lee SY, Kim J, Han J, Han MJ, An GH: Functional analyses of the flowering time gene OSMADS50, the putative SUPPRESSOR OF OVEREXPRESSION OF CO IIAGAMOUS-LIKE 20 (SOCI/AGL20) ortholog in rice. The Plant Journal 2004, 38:754-764.

4I. Petersen K, Kolmos E, Folling M, Salchert K, Storgaard M, Jensen CS, Didion T, Nielsen KK: Two MADS-box genes from perennial ryegrass are regulated by vernalization and involved in the floral transition. Physiologia Plantarum 2006, 126:268-278.

42. Shitsukawa N, Ikari C, Mitsuya T, Sakiyama T, Ishikawa A, Takumi S, Murai K: Wheat SOCI functions independently of WAPII $V R N I$, an integrator of vernalization and pnotoperiod flowering promotion pathways. Physiologia Plantarum 2007, 130:627-636.

43. $\mathrm{Ng} M$, Yanofsky MF: Function and evolution of the plant MADSbox gene family. Nature Reviews Genetics 200I, 2:186-195.

44. Ciaffi M, Paolacci AR, D'Aloisio E, Tanzarella OA, Porceddu E: Identification and characterization of gene sequences expressed in wheat spikelets at the heading stage. Gene 2005, 346:22I-230.

45. Murai $K$, Murai R, Ogihara Y: Wheat MADS box genes, a multigene family dispersed throughout the genome. Genes \& Genetic Systems 1997, 72:317-321.

46. Kane NA, Danyluk J, Tardif G, Ouellet F, Laliberte JF, Limin AE, Fowler DB, Sarhan F: TaVRT-2, a member of the StMADS-II clade of flowering repressors, is regulated by vernalization and photoperiod in wheat. Plant Physiology 2005, 138:2354-2363.

47. Limin AE, Fowler DB: Low-temperature tolerance and genetic potential in wheat (Triticum aestivum L.): response to photoperiod, vernalization and plant development. Planta 2006, 224:360-366.

48. De Bodt S, Raes J, Florquin K, Rombauts S, Pierre R, Theissen G, Peer $Y$ Van de: Genomewide Structural Annotation and Evolutionary Analysis of the Type I MADS-Box Genes in Plants. Journal of Molecular Evolution 2003, 56:573-586.

49. Zhao XY, Cheng Z], Zhang XS: Overexpression of TaMADS I, a SEPALLATA-like gene in wheat, causes early flowering and the abnormal development of floral organs in Arabidopsis. Planta 2006, 223:698-707.

50. Song LM, Shinyoung L, Hyo JK, Hong GN, An G: OsMADS5I is a short-day flowering promoter tht functions upstream of EhdI, OsMADSI4 and Hd3a. Plant Physiology 2007, I 45: | 484-I 494

51. Wilson ID, Barker GLA, Beswick RW, Shepherd SK, Lu CG, Coghill JA, Edwards D, Owen P, Lyon R, Parker JS, et al:: A transcriptomics resource for wheat functional genomics. Plant Biotechnology Journal 2004, 2:495-506.

52. Jensen WA: Botanical Histochemistry: Principles and Practice. San Francisco: W.H. Freeman; 1962. 